\title{
Violência infantil no Brasil: Panorama das notificações e indicadores desse fenômeno
}

\section{Child abuse in Brazil: overview of notifications and indicators of this phenomenon}

Fernanda Gonçalves da Silva ${ }^{1 *}$, Pâmela Cristine dos Santos Bastos da Fonseca ${ }^{2}$, Jeanne dos Santos Oliveira Marques Dantas ${ }^{2}$, Cíntia Cassimiro da Silva ${ }^{2}$, Clarissa Teixeira Cardoso de Carvalho ${ }^{2}$

\section{RESUMO}

Este estudo foi feito em duas etapas. A primeira atualizou o artigo intitulado "Violência infantil no Brasil e suas consequências psicológicas: uma revisão sistemática", estudo bibliográfico dos tipos de violência infantil mais estudados e suas consequências biopsicossociais como indicadores do fenômeno. A segunda analisou as notificações deste tipo de violência no Sistema de Informação de Agravos de Notificação (SINAN NET) e Instituto Brasileiro de Geografia e Estatística (IBGE), utilizando como critérios de análise a classificação dos tipos de violência da revisão anterior, a classificação etária e recorte temporal de acordo com a publicação do Estatuto da Criança e do Adolescente (ECA), 1990. A literatura identificou oito tipos de violência, destacando a sexual. Foram identificados diversos indicadores, sendo evidenciados: os danos psicológicos/emocionais, traumas/doenças físicas, abuso de álcool e outras substâncias, problemas no desenvolvimento, entre outros. Em contraste, o SINAN NET, que notifica compulsoriamente, destacou a negligência/abandono, seguida da violência física. Já o IBGE, que levanta dados sociodemográficos, apresentou informações mais generalistas e escassas.

Palavras-chave: Maus-tratos infantis; Notificação de abuso; Proteção da criança; Saúde da criança; Indicadores (Estatística).

\begin{abstract}
This study was done in two stages. The first stage updated the study entitled "Child violence in Brazil and its psychological consequences: a systematic review", a bibliographical study of the most studied types of child violence and its biopsychosocial consequences as indicators of the phenomenon. The second stage analyzed the notifications of this type of violence in Sistema de Informação de Agravos de Notificação (SINAN NET) and Instituto Brasileiro de Geografia e Estatística (IBGE), using as analysis criteria the classification of types of violence from the previous review, the age and year classification according to the publication of the Estatuto da Criança e do Adolescente (ECA), 1990. The literature identified eight types of violence, highlighting sexual violence. Several indicators were highlighted: psychological/emotional damage, trauma/physical illness, alcohol and other substance abuse, developmental problems, among others ones. In contrast, SINAN NET, which reports compulsory, highlighted negligence/abandonment, followed by physical violence. The IBGE, which surveys sociodemographic data, presented more generalist and scarcer information.
\end{abstract}

\footnotetext{
${ }^{1}$ Universidade Federal do Rio de Janeiro 1. *E-mail: fernandagoncalves.fgs@ gmail.com 2 Universiddade Estácio de Sá
} 
Keywords: Child abuse; Mandatory reporting; Child welfare; Child health; Indicators (Statistics).

\section{INTRODUÇÃO}

Violência consiste no uso intencional da força física ou do poder, de forma real ou em ameaça, que resulte ou tenha grande possibilidade de resultar em lesão, morte, dano psicológico, desenvolvimento ou privação. Este conceito abarca as mais diversas tipologias de violência, desde agressão física à violência psicológica. Assim, a violência é caracterizada como um dos principais problemas mundiais de saúde pública, segundo a Organização Mundial da Saúde, conforme o Relatório Mundial sobre Violência e Saúde (OMS, 2002).

Diversos fatores interagem na problemática da violência infantil, pois trata-se de uma população vulnerável nos aspectos físico, emocional, cognitivo e social. O processo de desenvolvimento da criança é predominantemente influenciado pelo meio no qual está inserida, provendo aspectos propícios para o seu crescimento ou inferindo condições desfavoráveis. Estas condições se configuram de diversas maneiras, sendo a violência uma delas. (SILVA et al., 2018). De acordo com o Estatuto da Criança e do Adolescente (ECA, 2008), a violência infantil é um fenômeno multifacetado, incluindo outras maneiras apresentação como o bullying, a alienação parental e o trabalho infantil.

Segundo o relatório Status report on preventing violence against, a OMS tem como meta a extinção da violência contra crianças em até 2030, promovendo estratégias mundiais para alcançar tal objetivo, já que esta atinge até um bilhão de crianças ao redor do mundo. A mensuração frequente das notificações permitirá ações mais contundentes para o cumprimento das metas de redução desse fenômeno. (OMS, 2020).

Criança é a pessoa com até 12 anos de idade incompletos e adolescente, aquela entre 12 e 18 anos de idade. Ambas gozam de direitos fundamentais, tais como o direito à vida, saúde, à liberdade, ao respeito, dignidade, à convivência familiar e comunitária. As oportunidades e facilidades devem lhe ser asseguradas a fim de lhes facultar o desenvolvimento físico, mental, moral, espiritual e social (ECA, 2008).

Desta maneira, toda criança deve ter seu pleno de desenvolvimento, ou seja, longe de qualquer tipo de castigo físico, de tratamento cruel ou condição degradante, por parte de seus responsáveis legais. Estes possuem o dever de cuidar e zelar por uma educação digna, com proteção, sem utilizar de medidas punitivas, com pretextos de correção. Assim 
configura-se a violência física infantil toda atitude intencional de ferir ou causar dor na criança, desdobrando-se de forma fatal ou não. (ECA, 2008; UNICEF, 2006).

É relevante ressaltar que as diferentes formas de violência infantil podem ocorrer concomitantemente, propiciando intensos impactos à vítima. Quanto mais rapidamente for percebida a violência, as possibilidades de intervenções e ressignificações se tornam mais viáveis. Para isso, é necessário que se tenha cada vez mais informações sobre o fenômeno e suas consequências à vítima, já que isso possibilitará a sua identificação, uma vez que essas consequências podem ser servir como indicadores deste problema social.

Se por um lado é dever da família, da sociedade e do Estado garantir os direitos e a proteção integral da criança, por outro lado é evidente que existem profissionais de saúde que se encontram despreparados para a identificação da violência. Além disso, há uma carência de apoio para encaminhamento dos casos, o que favorece o silenciamento da violência. Este fato pode estar relacionado a inúmeros fatores, como a cultura, o receio de denunciar, desinformação, entre outros (QUADROS et al., 2016).

Diante da magnitude do fenômeno, este estudo objetivou fazer uma análise das notificações da violência infantil no Brasil e de seus indicadores. Desta maneira, o estudo pode contribuir para o esclarecimento deste tipo de violência através de informações sobre as características e proporção deste problema que impacta a saúde pública direta e indiretamente.

\section{MÉTODO}

O presente estudo foi realizado em duas etapas. A primeira se dedicou a fazer atualização de um estudo de revisão sistemática já publicada e intitulada "Violência infantil no Brasil e suas consequências psicológicas: uma revisão sistemática”. A segunda se propôs a fazer uma análise dos casos de violência infantil com base em dados do Instituto Brasileiro de Geografia e Estatística (IBGE) e dados do Sistema de Informação de Agravos de Notificação (SINAN NET).

Assim, foi possível realizar uma análise dos tipos de violência aos quais os estudos científicos se dedicam mais a pesquisar em comparação aos tipos de violência que são notificados compulsoriamente no SINAN NET. Desta maneira, foi possível verificar se há uma concordância entre o que está sendo estudado e os dados notificados nas bases.

\section{Revisão sistemática}


Para a continuidade à revisão sistemática foram utilizadas as bases de dados PubMed e SciELO, como no estudo-base. Os critérios de elegibilidade dos artigos foram: corresponder ao tema violência infantil através dos descritores: "child abuse", "child abuse and Brazil", "violence against child", "psychological effects of child abuse in Brazil" e "consequences of child abuse in Brazil", apresentar ao menos uma consequência deste fenômeno e estar nos idiomas: inglês, português ou espanhol. Além disso, os estudos também deveriam datar a partir de 1990, quando ocorreu a publicação da Lei 8.069/90 que dispõe do ECA.

Esta etapa seguiu basicamente a metodologia do estudo-base, porém seu recorte temporal foi diferente, propondo, então, uma atualização dos dados obtidos. O estudobase também utilizou o recorte temporal da promulgação do ECA, 1990, finalizando as buscas em fevereiro de 2019, logo, esta atualização compreende o período posterior a esse, sendo finalizada no dia 30 de novembro de 2020.

\section{Análise dos casos de violência infantil}

A coleta de dados para a análise dos casos de violência de ambas as bases, IBGE e SINAN NET, ocorreu seguindo os mesmos critérios: as classificações dos tipos de violência infantil segundo o artigo-base e classificação etária do ECA. O recorte temporal também seguiu o critério da criação do ECA. O acesso à base do SINAN NET ocorreu via DATA SUS.

\section{RESULTADOS}

\section{Revisão Sistemática}

$\mathrm{Na}$ atualização da revisão sistemática, foram encontrados 294 artigos, dos quais 39 foram excluídos por repetição, 115 foram excluídos por título, 64 por resumo, nenhum por idioma, 55 por leitura na íntegra e 11 por estarem indisponíveis para acesso. Sendo selecionados então, 10 artigos para análise e extração de dados.

\section{Tipos de violência mais identificados segundo a literatura}

Sobre os tipos de violência infantil, foram identificados oito conforme mostra o gráfico 1, sendo a violência sexual o tipo mais comumente estudado pela literatura, dado que destaca a violência sexual em detrimento da violência física. No estudo primário tanto a violência sexual como a física obtinham prevalência nas pesquisas cientificas analisadas. 
O estudo-base contou com 36 artigos selecionados, que somados aos 10 deste estudo atual totalizaram 46. Porém é possível observar que os números do gráfico abaixo totalizam mais do que 46. Isso se dá ao fato de que, geralmente, os artigos se dedicam a estudar mais de um tipo de violência infantil.

Gráfico 1 - Tipos de violências mais identificadas na literatura

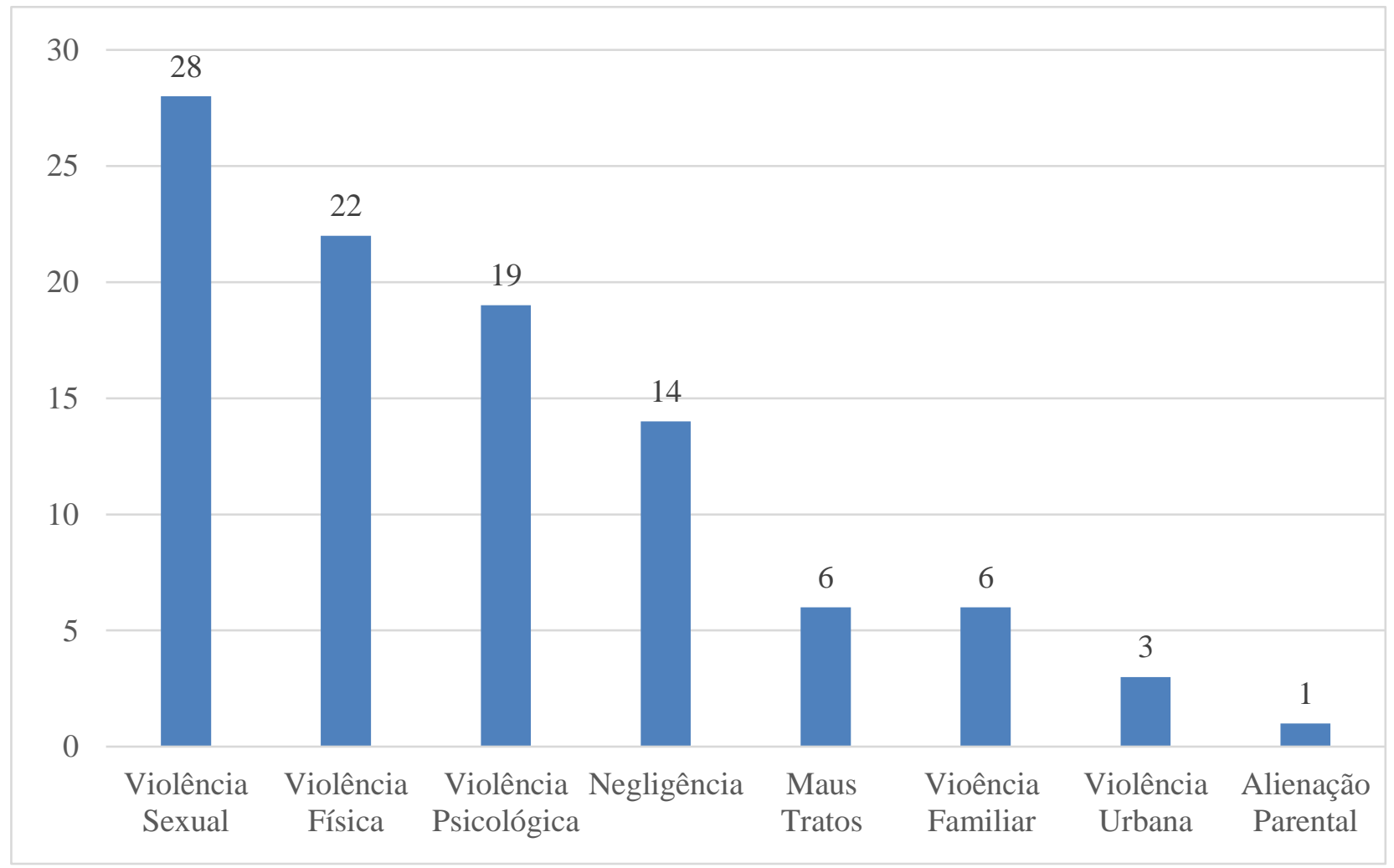

\section{Consequências e indicadores da violência infantil segundo a literatura}

É possível identificar diversas consequências biopsicossociais como indicadores da violência infantil na literatura. Esses indicadores podem ser de diferentes ordens que podem variar de acordo com o tipo de violência sofrida. Porém, alguns deles possuem maiores índices independentemente do tipo de violência sofrida, como os danos psicológicos e/ou emocionais, danos sociais, traumas e doenças físicas, além do abuso do uso de álcool e substâncias, como mostra o gráfico 2 a seguir. 
Gráfico 2 - Indicadores da violência infantil de acordo com a literatura

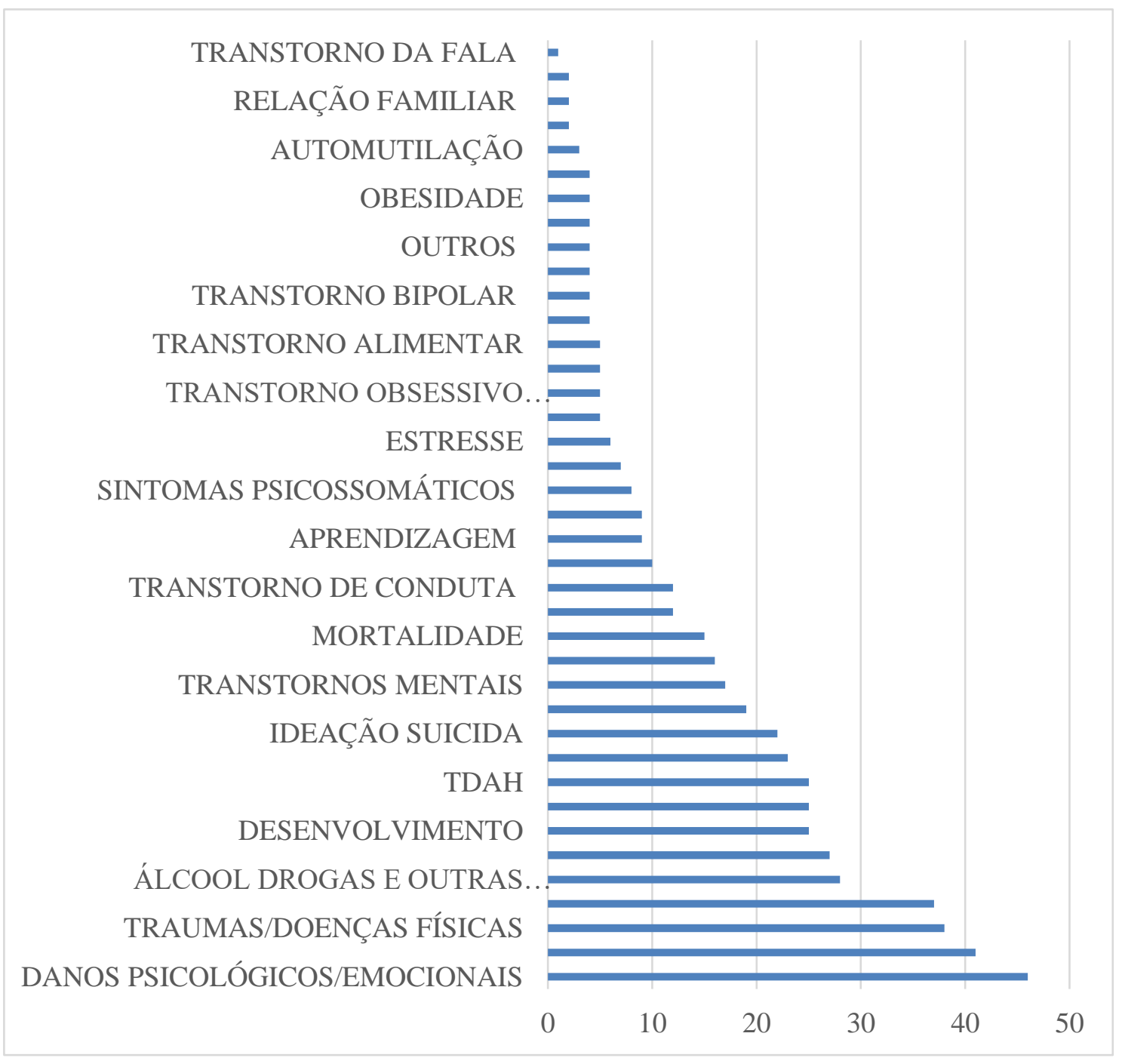

\section{Base do IBGE}

A coleta de dados nesta base foi realizada através das informações disponíveis a respeito de cada série cadastrada que se referia à população infantil segundo a classificação etária do ECA até 12 anos de idade. Porém, por não haver menções claras à violência infantil, foi necessário classificar as séries de acordo com o tipo de violência que elas representavam. Assim, os tipos identificados foram: negligência, violência urbana, maus-tratos, trabalho infantil ou outros tipos de violência, podendo cada série representar mais de um destes tipos. 
Tabela 1 - Dados de violência infantil na base IBGE

\begin{tabular}{|c|c|c|c|c|c|}
\hline Nome da série cadastrada & Período & Dados relativos & $\begin{array}{l}\text { Unidade } \\
\text { de medida }\end{array}$ & $\begin{array}{l}\text { Tipo de } \\
\text { violência }\end{array}$ & Eixo \\
\hline $\begin{array}{l}\text { Pessoas de } 5 \text { anos ou mais } \\
\text { de idade não alfabetizadas, } \\
\text { por grupos de idade }\end{array}$ & $\begin{array}{l}2001- \\
2015\end{array}$ & $\begin{array}{l}5-6 \text { anos: } 2001: 3,15 \\
\text { 2015: } 1,72 \\
7 \text { anos: } 2001: 0,68 \\
\text { 2015: } 0,29 \\
8-9 \text { anos: } 2001: 0,58 \\
\text { 2015: } 0,22 \\
\text { 10 -11 anos: } 2001: 0,25 \\
\text { 2015: } 0,07 \\
\text { 12 anos: } 2001: 0,08 \\
\text { 2015: } 0,02\end{array}$ & Percentual & Negligência & $\begin{array}{l}\text { Taxa de } \\
\text { analfabeti } \\
\text { smo }\end{array}$ \\
\hline $\begin{array}{l}\text { Taxa de analfabetismo, por } \\
\text { grupos de idade }\end{array}$ & $\begin{array}{l}1992- \\
2011\end{array}$ & $\begin{array}{l}10-14 \text { anos: } 1992: 12,4 \\
2011: 1,9\end{array}$ & Percentual & Negligência & $\begin{array}{l}\text { Taxa de } \\
\text { analfabeti } \\
\text { smo }\end{array}$ \\
\hline Taxa de mortalidade infantil & $\begin{array}{l}1997- \\
2008\end{array}$ & $\begin{array}{l}\text { 1997: } 31,9 \\
2008: 17,56\end{array}$ & $\begin{array}{l}\text { Por } 1000 \\
\text { nascidos } \\
\text { vivos }\end{array}$ & $\begin{array}{l}\text { Negligência } \\
\text { ou outros } \\
\text { tipos de } \\
\text { violência }\end{array}$ & $\begin{array}{l}\text { Mortalida } \\
\text { de } \\
\text { infantil }\end{array}$ \\
\hline $\begin{array}{l}\text { Óbitos mal definidos de } \\
\text { menores de } 1 \text { anos de idade } \\
\text { - Mortalidade proporcional } \\
\text { por causas }\end{array}$ & $\begin{array}{l}1990- \\
2007\end{array}$ & $\begin{array}{l}\text { 1990: } 19,2 \\
\text { 2007: } 6,9\end{array}$ & Percentual & $\begin{array}{l}\text { Negligência } \\
\text { ou outros } \\
\text { tipos de } \\
\text { violência }\end{array}$ & $\begin{array}{l}\text { Mortalida } \\
\text { de } \\
\text { infantil }\end{array}$ \\
\hline $\begin{array}{l}\text { Óbitos sem assistência } \\
\text { médica de menores de } 1 \text { ano } \\
\text { de idade }\end{array}$ & $\begin{array}{l}1990- \\
2004\end{array}$ & $\begin{array}{l}\text { 1990: } 16,6 \\
2004: 3,7\end{array}$ & Percentual & Negligência & $\begin{array}{l}\text { Mortalida } \\
\text { de } \\
\text { infantil }\end{array}$ \\
\hline $\begin{array}{l}\text { Óbitos por doença diarreica } \\
\text { aguda - menores de } 5 \text { anos - } \\
\text { Mortalidade proporcional }\end{array}$ & $\begin{array}{l}1990- \\
2009\end{array}$ & $\begin{array}{l}\text { 1990: } 10,8 \\
2009: 2,5\end{array}$ & Percentual & Negligência & $\begin{array}{l}\text { Mortalida } \\
\text { de } \\
\text { infantil }\end{array}$ \\
\hline $\begin{array}{l}\text { Taxa de mortalidade } \\
\text { específica por AIDS - } \\
\text { crianças menores de } 13 \text { anos }\end{array}$ & $\begin{array}{l}1990- \\
2009\end{array}$ & $\begin{array}{l}\text { 1990: } 0,7 \\
\text { 2009: } 0,2\end{array}$ & $\begin{array}{l}\text { Óbitos } \\
\text { por } 100 \\
\text { mil hab }\end{array}$ & Negligência & $\begin{array}{l}\text { Mortalida } \\
\text { de } \\
\text { infantil }\end{array}$ \\
\hline Taxa de Trabalho Infantil & $\begin{array}{l}1992- \\
2008\end{array}$ & $\begin{array}{l}\text { 1992: } 23,63 \\
\text { 2008: } 9,56\end{array}$ & Percentual & $\begin{array}{l}\text { Violência } \\
\text { urbana/ } \\
\text { Maus-tratos }\end{array}$ & $\begin{array}{l}\text { Trabalho } \\
\text { infantil }\end{array}$ \\
\hline $\begin{array}{l}\text { Taxa de trabalho infantil no } \\
\text { Brasil metropolitano }\end{array}$ & $\begin{array}{l}1992- \\
2008\end{array}$ & $\begin{array}{l}\text { 1992: } 10 \\
\text { 2008: } 4,22\end{array}$ & Percentual & $\begin{array}{l}\text { Violência } \\
\text { urbana/ } \\
\text { Maus-tratos }\end{array}$ & $\begin{array}{l}\text { Trabalho } \\
\text { infantil }\end{array}$ \\
\hline
\end{tabular}


Nível de ocupação de crianças de 5 a 9 anos de idade, por sexo
1992 -

1995 e

1998 -

2007

Nível de ocupação de crianças e adolescentes de 5 a 17 anos de idade, por sexo
1992 -

$1995 \mathrm{e}$

1998 -

2007
Total: $1992: 3,67$

2007: 0,87

Sexo feminino: 1992:

2,13

2007: 0,54

Sexo masculino: 1992:

5,17

2007: 1,19

Total: 1992:19,55

2007: 10,6

Homens: 1992: 25,36

2007: 13,52

Mulheres: 1992:13,64

2007: 7,53

1992 - Atividade agrícola:

1995 e $1992: 49,5$

1998 - 2007: 37,5

2007 Atividade não agrícola:

1992: 50,5

2007: 62,5

1992 -

Atividade agrícola:

1995 e $1992: 39,5$

1998 - 2007: 25,6

2007 Atividade não agrícola:

1992: 60,5

2007: 74,4

1992 - Atividade agrícola:

1995 e $\quad 1992: 54,8$

1998 - 2007: 43,7

2007 Atividade não agrícola:

1992: 45,2

2007: 56,3

Crianças e adolescentes de

5 a 17 anos de idade,

ocupadas na atividade não

agrícola - Trabalho

principal

Crianças e adolescentes de

5 a 17 anos de idade,

ocupadas na atividade

agrícola - Trabalho

principal

1992 - $\quad 5$ a 9 anos: 1992: 18,39

1995 e 2007: 27,57

1998 - $\quad 10$ a 14 anos: 1992:

$2007 \quad 40,75$

2007: 46,82

1992 - $\quad 5$ a 9 anos: 1992: 81,61

1995 e 2007: 72,43

1998 - $\quad 10$ a 14 anos: 1992:
59,25

2007: 53,18
Percentual Violência urbana/ Maus-tratos

Trabalho infantil

Percentual Violência urbana/

Trabalho infantil

Maus-tratos

Percentual Trabalho

Trabalho infantil infantil
Percentual Trabalho
Trabalho infantil infantil

Percentual Trabalho

Trabalho infantil infantil

Percentual Trabalho

Trabalho infantil infantil

Percentual Trabalho Trabalho Infantil infantil 
Como é possível observar na Tabela 1, há uma tendência geral de decréscimo dos casos de violência infantil, presente em 12 das 16 séries analisadas, com exceção das séries referentes às crianças e adolescentes na ocupação agrícola e não agrícola, ou seja, dados relativos ao trabalho infantil.

Essas séries que apresentaram aumento possuem algumas características comuns que permitem fazer a inferência de que seus dados são oriundos de uma mesma pesquisa que foi dividida em séries para ser analisada. Ambas as séries utilizaram a unidade de medida percentual, foram realizadas entre o período de 1992 e 1995 e tiveram uma pausa de dois anos, retomando a coleta de dados entre 1998 e 2007.

Além disso, também abordam a mesma faixa etária, de cinco a dezessete anos, expondo tendência de aumento dos casos de trabalho infantil na atividade não-agrícola. No entanto, esses dados se contrastam com as informações das séries oito e nove realizadas entre o período de 1992 e 2008 , que englobam o intervalo das pesquisas em questão e relatam claramente um decréscimo do trabalho infantil.

\section{Base SINAN NET}

Esta base mostrou dados diferentes da literatura, evidenciando a negligência/abandono (29\%) e a violência física (28\%), seguidas da violência sexual (16\%), que também demonstrou percentual significativo em relação aos demais tipos de violência identificados, como é exposto no gráfico 3.

Gráfico 3 - Prevalência das violências notificadas no SINAN NET

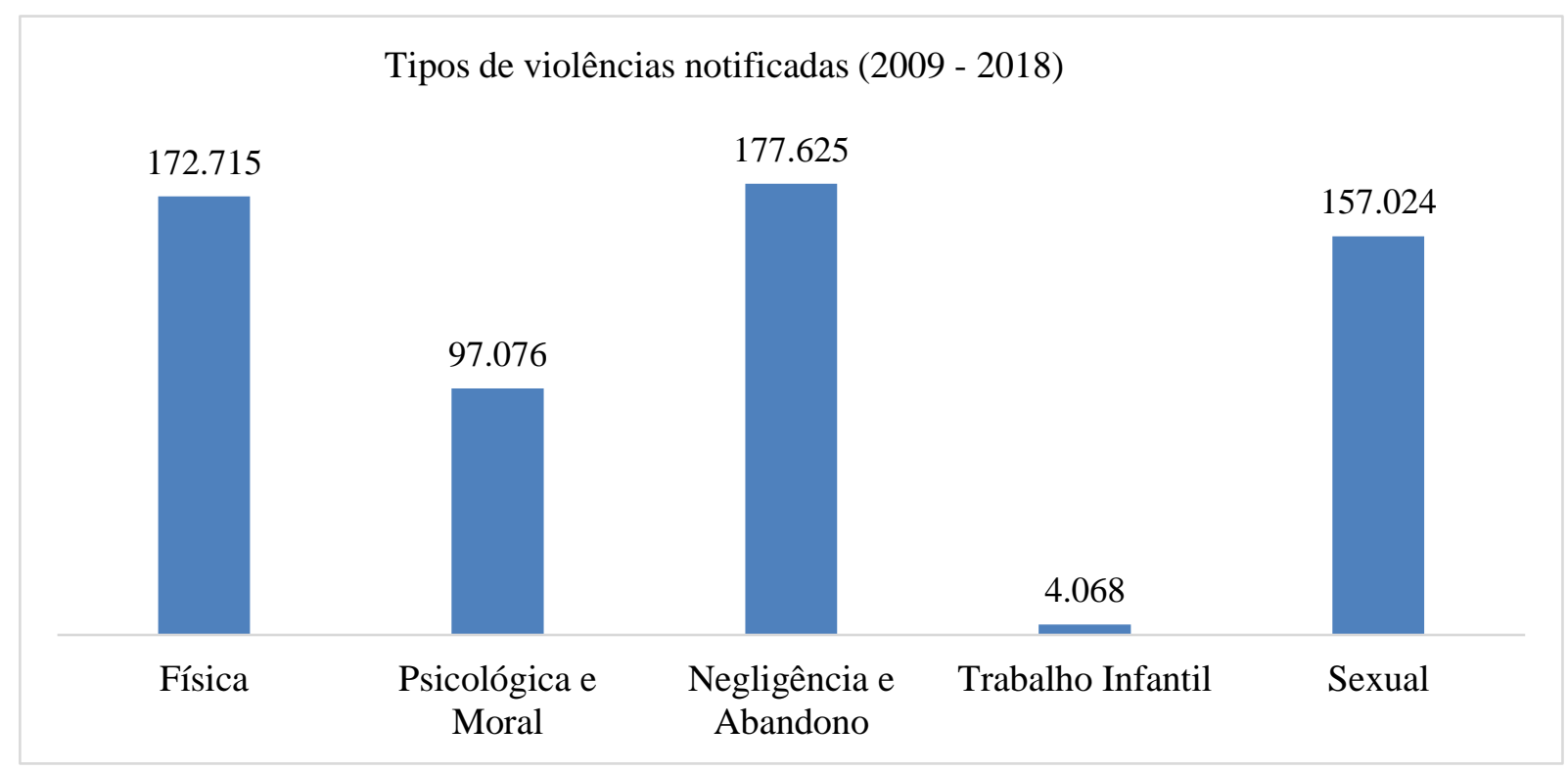


A referida base ainda faz uma classificação de acordo com a incidência de cada tipo de violência por idade da vítima. Assim, destacou-se a prevalência da violência física $(51,36 \%)$, e psicológica e moral $(48,85 \%)$, possuem maior incidência na faixa etária entre dez e catorze anos. Por outro lado, negligência e abandono, tem maior impacto em crianças entre um e quatro anos, afetando 38,19\% das vítimas. Em relação ao trabalho infantil, 66,03\% das vítimas possuem idades entre dez e catorze anos. Em casos de violência sexual verifica-se maior número de notificações nesta mesma faixa etária (48,76\%), porém também há incidências relevantes com vítimas da primeira infância, com $20,2 \%$ dos casos na faixa de um a quatro anos e $28,9 \%$ na faixa de cinco a nove anos.

É possível observar no gráfico 4 a seguir que há uma tendência de aumento progressivo das notificações de violência infantil no Brasil. O gráfico mostra que em menos de dez anos, o número de casos notificados aumentou quase seis vezes em relação ao ano inicial da pesquisa. Porém, este número ainda pode ser superior devido às subnotificações.

Gráfico 4 - Evolução do total de notificações no SINAN NET no período entre 2009 e 2018

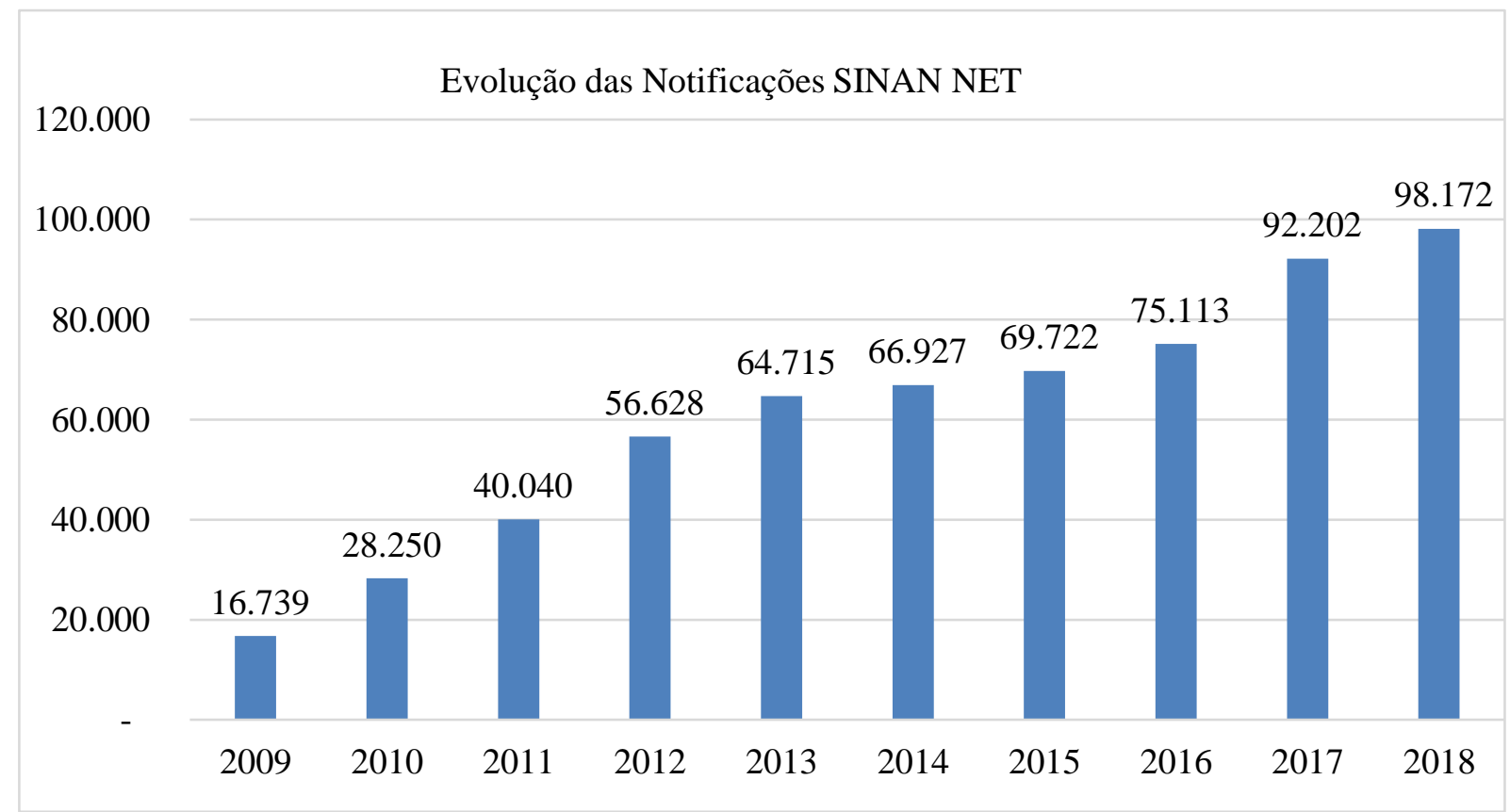

DISCUSSÃO

IBGE e SINAN 
Ao analisar as bases IBGE e SINAN NET, algumas objeções referentes à extração de dados e classificação dos tipos de violência foram identificadas. A base IBGE não dispõe de informações detalhadas acerca da violência infantil. As pesquisas são realizadas através de um censo da população, possuindo um viés mais sociodemográfico e evidenciando questões sociais de maneira mais generalista tal como o analfabetismo, mortalidade infantil, trabalho infantil entre outras. Além disso, as séries não possuíam um recorte temporal padrão, bem como algumas delas omitiam algum ano ao longo do período pesquisado.

A unidade de medida utilizada pela referida base para contabilizar os dados relativos também variou, sendo algumas séries analisadas segundo a medida percentual e outras por número de pessoas, o que impossibilitou uma análise comparativa entre essas séries. Então, a análise foi realizada através de uma comparação entre os dados da própria série. Para isso, o ano inicial da pesquisa de cada série e seu respectivo ano final foram comparados a fim de verificar se houve um aumento ou declínio dos casos contabilizados pelas séries e, consequentemente, de cada tipo de violência infantil.

O que ficou evidente nesta classificação é que houve um decréscimo dos casos de violência infantil. Isso foi observado em doze das dezesseis séries analisadas, exceto nas séries que citam a ocupação agrícola, dados relativos ao trabalho infantil. Estes dados sugerem que há carência de políticas públicas sociais e assistenciais do Brasil. Ao observar as séries que abordam o analfabetismo e a mortalidade por HIV-AIDS em indivíduos menores de 13 anos, foi possível verificar também que o tipo de violência a qual foram classificadas essas séries corresponde à negligência, explicitando que nesses casos o Estado configura-se como um potencial agressor.

Quanto aos dados coletados pelo SINAN NET, por se tratar de uma notificação compulsória, esses são agrupados por faixa etária, respeitando os limites de idade da infância, estipulados pelo ECA. Foi possível identificar quatro faixas etárias: menores de um ano, de um a quatro anos, cinco a nove anos e de 10 a 14 anos, o que facilitou a identificação dos grupos de maior volume de notificações por tipo de violência. Caberá um novo estudo para avaliar detalhamento da incidência de determinados tipos de notificações das violências em determinadas faixas etárias.

Os estudos científicos, então, abordam mais tipos de violência infantil do que a base de notificação compulsória, no entanto, os dados percentuais não são tão 
discrepantes. Ambos destacam a violência sexual, a física e a psicológica, com $67 \%$ dos estudos na literatura e $70 \%$ das notificações.

Entretanto, no SINAN NET, o tipo negligência/abandono $(29,19 \%)$ sobrepõe todas as formas violências, mas com percentual próximo a segunda mais comum, que foi a violência física (28,38\%). Pode-se inferir que, em função das notificações serem feitas por profissionais da saúde, violências que deixam sinais corporais evidentes possibilitam sua identificação mais comumente, através dos indicadores do fenômeno.

\section{Indicadores}

Como podemos observar, as violências produzem consequências biopsicossociais que impactam o desenvolvimento infantil. Essas consequências podem servir de indicadores desse fenômeno ao permitir a identificação de aspectos comuns entre indivíduos que tenham vivenciado tais eventos, sendo entendidos como resultados dessas vivências. Tais indicadores surgem da violência experenciada e de acordo com o tempo durante o qual ocorre, podendo ser projetados no decorrer de toda a vida do sujeito (NUNES et al., 2020).

A utilização de castigos severos ou a postura relapsa dos responsáveis de maneira omissa e negligente, faltando com atenção e afeto necessários para vida da criança, podem ser pontos fundamentais para o desenvolvimento de transtornos de comportamento, como os de conduta. Estes tipos de transtornos podem ser expressos pelo vandalismo, roubo, entre outros. (BORDIN et al., 2009).

A violência sexual sofrida pode afetar diretamente as relações interpessoais ao longo da vida da vítima. Ansiedade intensa, insegurança, baixa autoestima, desconfiança das pessoas de seu convívio e comportamentos sexuais não apropriados para a idade são indicadores dessa experiência (REICHENHIN et al., 1999, RIBEIRO et al., 2004).

A tentativa de suicídio ou comportamento suicida, que compreende a automutilação ou lesão autoprovocada, destaca-se como consequências subsequentes do abuso sexual infantil, que são mais recorrentes em vítimas que sofreram abuso com penetração. Essas vítimas são mais propensas à gravidez indesejada, abortamento e à contração doenças infecciosas.

O desenvolvimento de transtornos mentais ou de comportamento trazem respostas do impacto desses fenômenos, por se tratar de uma violência praticada, principalmente, por pais, padrasto, tios, avós ou primos. Portanto, pessoas com as quais as vítimas 
possuem vínculos afetivos e convívio constante, antes, durante e depois da ocorrência do abuso.

Verifica-se também o transtorno de estresse pós-traumático (TEPT) presente nessas vítimas (PLATT et al., 2018). O TEPT, a depressão e a ansiedade podem ser desdobramentos de experiências mais traumatizantes de violência sexual e física, apresentando-se em outros tipos de violência, no entanto com notificações menos expressivas.

Passar por essas experiências traumatizantes pode interferir na maneira como essas crianças enxergam o mundo, afetando os seus pensamentos, corroborando para o reforço dos sintomas destes transtornos. Desta maneira, o medo de vivenciar novamente esses traumas e o sentimento de culpa impactam no convívio social e nas relações interpessoais. (HABIGZANG et al.,2009).

Transtornos mentais comuns como ansiedade, depressão e somatização estão também diretamente relacionados às vítimas de abuso infantil e negligência. Esses transtornos mentais podem interferir no desenvolvimento e na formulação da personalidade da vítima.

A violência psicológica interfere negativamente na construção da resiliência dessas vítimas e todas as vertentes do desenvolvimento humano. No entanto, constatouse que vítimas que recebiam expressões de amor e afeto dentro do ambiente familiar, advindo dos cuidadores foi um preditor para redução do agravamento dessas consequências. (MORAES et al., 2018).

O transtorno de déficit atenção e hiperatividade (TDAH) também se apresentou como um indicador de violência infantil. A criança que vive em um ambiente familiar hostil, presenciando frequentes brigas, sendo vítima de agressões físicas e verbais, pode apresentar o TDAH.

Consequentemente, isso pode desencadear o aumento da utilização da força física e castigos severos por parte dos responsáveis a fim de "corrigir" tal transtorno. Logo, isso propicia uma rotina cada vez mais violenta, pelo fato dos responsáveis frequentemente não obterem habilidades para relacionar-se com seus filhos (PIRES et al., 2012).

A violência familiar integra diversos indicadores potencializadores de distúrbios do sono, aumentando a propensão à utilização de álcool, fumo e drogas ilícitas nas faixas etárias vindouras. Tentativas de suicídio podem se apresentar de forma recorrente, além de agressividade, isolamento e desinteresse alimentar (REICHENHIN et al., 1999). 
Além disso, vivenciar a violência no âmbito familiar acarreta prejuízos caracterizados por conflitos, sentimentos de inadequação e culpa. As relações familiares podem se romper por completo em consequência da quebra dos vínculos afetivos e por determinações judiciais. (FERREIRA et al., 2019).

Outros indicadores foram apontados como transtornos de eliminação, transtorno opositor desafiador - TOD, transtorno de conduta, fobias, estresse agudo, entre outros. Estes problemas de saúde mental ocorrem em indivíduos que vivenciaram violência urbana, negligência, violência física e emocional, no lugar de um vínculo seguro, de um ambiente familiar favorável e propiciador de uma infância saudável (HOFFMANN et al., 2016).

Diversos são os desdobramentos de experienciar a violência na infância. Não foi possível mensurar o quanto essas vítimas serão impactadas por cada tipo de violência. Isso dependeria de outros aspectos relacionados a essa experiência e a outras variáveis que não foram identificadas neste estudo. Portanto, se faz necessário investigar a correlação dessas consequências com outras variáveis. Porém, é pertinente ressaltar que os indicadores podem auxiliar profissionais de saúde, educação e pessoas do convívio das vítimas de violência a identificar e interceptar possíveis ocorrências.

\section{Tipos de violência}

A violência sexual infantil alcançou cerca de 157.024 vítimas durante o período entre 2009 e 2018. Este tipo é mais prevalente em vítimas de 10 a 14 anos com 48,76\% dos casos notificados segundo SINAN NET. É relevante destacar que apesar da violência sexual ser a mais pesquisada na literatura (NUNES et al., 2020), quando mensuradas as notificações, verificou-se uma divergência. A violência sexual foi a terceira mais notificada.

A violência psicológica, em função de frequentemente ser pesquisada em conjunto com outras violências, pode promover o entendimento errôneo de que não se apresenta sozinha. No entanto, este tipo de violência compreende cerca de 97.076 dos casos notificados pelo SINAN NET durante o período analisado, se configurando como $16 \%$ do total das notificações. Este dado evidencia sua ocorrência nos lares, atingindo principalmente a população da faixa etária de 10 a 14 anos.

Por não produzir marcas visíveis, é possível que muitas crianças experienciem a violência psicológica sem que esta seja notificada por profissionais de saúde. Isso pode 
ocorrer em função da dificuldade de sua identificação, o que permite a inferência de que esse número está aquém da real abrangência da situação.

No IBGE, as classificações de negligência nas séries de analfabetismo e taxas de mortalidade infantil no período aproximado de 1990 a 2015 tiveram um declínio ao longo dos anos. O SINAN NET apontou que a negligência/abandono atinge certa de 177.625 dos casos, sendo prevalência nas notificações de violência infantil. Vítimas com idades de um a quatro anos são as mais comuns nessas intercorrências, com 38,19\% das notificações.

A primeira infância é primordial para um desenvolvimento saudável. Logo, essa faixa etária é a de maior vulnerabilidade, pois é a que mais necessita de vínculos seguros para seu desenvolvimento. No entanto, foi observado que, apesar da negligência/abandono ser o tipo mais notificado, existem poucas pesquisas que se propõem a compreender os fatores relacionados a essas ocorrências. Assim, é necessária a realização de estudos subsequentes que se aprofundem nesse tópico (ECA, 1990, MACEDO, 2020).

Nas séries cadastradas da base de dados IBGE é possível verificar que a atividade agrícola e não agrícola caracterizam o trabalho infantil. Tais séries exibem números que evidenciam um aumento dos casos em crianças e adolescentes de cinco a 17 anos entre os anos de 1992 a 1995. Há ainda notificações de violências trazidas pelo SINAN NET que contabilizam 4.068 casos de trabalho infantil nos anos de 2009 a 2018.

No estudo anterior esses aspectos foram pouco delineados (NUNES et al., 2020). Os dados eram secundários, mais generalistas, além de utilizar uma nomenclatura diferente para descrever este tipo de violência, que apareceu como violência urbana, evidenciando a violência no contexto das cidades (NUNES et al., 2020). A UNICEF (2006) explica que, no Brasil, a violência em que a criança ou adolescente são submetidos não se difere em áreas rurais e urbanas.

Ao analisar os dados da literatura e da base SINAN NET, foi possível verificar que os recortes temporais das informações disponíveis divergem. Os estudos científicos possuem uma amplitude maior a partir do ano de promulgação da legislação do ECA, 1990, relatando dados entre 1992 e 2020. Já o SINAN NET dispõe apenas de dados mais recentes, entre 2009 e 2018 . Esta disparidade entre os intervalos de levantamento de dados pode explicar os resultados distintos a respeito das modalidades de violência. 
Dentre os tipos de violência infantil que a literatura aponta temos, foi possível verificar um número pouco expressivo de menções a violência familiar, que aparece em seis dos 46 artigos que compõem esse estudo. Além dessa modalidade, a alienação parental também se apresentou de maneira menos significativa, em apenas um dos referidos artigos. Tais violências não foram contempladas nas notificações do IBGE e SINAN NET.

Em um levantamento de dados da base SINAN NET foi possível observar uma tendência progressiva de aumento da violência. Em menos de 10 anos verificou-se que as notificações aumentaram seis vezes em relação ao ano inicial da pesquisa, se configurando como um problema social alarmante. É relevante destacar que esses números podem ser ainda maiores devido às subnotificações.

No contexto dessas subnotificações podemos inferir alguns aspectos que podem contribuir para a manutenção do fenômeno. Dentre estes aspectos estão a naturalização da violência, assim como a falta de preparo e instrução sobre os direitos e proteção da criança e do adolescente por parte dos profissionais da atenção primária da saúde.

Um estudo de Maceió, expõe notificações de nascidos vivos nos anos de 2009 a 2017, trazendo um registro de 953 nascimentos de crianças com mães com idades entre 10 e 13 anos. Desses casos, somente 1,3\% foram notificados como abuso sexual, outros 20,3\% declararam ser casadas ou em união estável (SILVA et al., 2020).

Os dados evidenciam a naturalização da gravidez e do casamento neste grupo. Os profissionais de saúde, não percebendo a vulnerabilidade e adultização dessas meninas, deixam de notificar tal violência, desconhecendo ou desconsiderando as consequências que o feito pode acarretar (SILVA et al., 2020).

Portanto, se torna evidente a precarização de condições de trabalho e a falha na empregabilidade de políticas de saúde pública eficazes que garantam o bem-estar da população e dos profissionais da saúde. Assim, pode-se inferir que a falta de estrutura e conhecimento dos desdobramentos da violência infantil podem dificultar sua identificação e logo notificação dos casos de violência que chegam à assistência de saúde básica.

Isso evidencia a necessidade de programas de capacitação desses profissionais, através de ações multidisciplinares e interdisciplinares a fim de oferecer à população uma equipe mais qualificada. Novos estudos são sugeridos para verificar possíveis variações 
de dados a nível regional, bem como a eficácia de ações locais que visem a mitigação dos riscos de violência infantil.

\section{REFERÊNCIAS}

BORDIN, I. A. S. et al. Severe physical punishment: risk of mental health problems for poor urban children in Brazil. Bull World Health Organ, s./l., n. 87, v. 5, p. 366-344, may 2009. DOI: https://doi.org/10.2471/BLT.07.043125. Available on:

<https://www.ncbi.nlm.nih.gov/pmc/articles/PMC2678773/>. Access in: 25 apr. 2021.

BRASIL. Lei no 8.069, de 13 de julho de 1990. Dispõe sobre o Estatuto da Criança e do Adolescente e dá outras providências. Diário Oficial [da] República Federativa do Brasil, Brasília, DF, 13 jul. 1999. Disponível em:

<http://www.planalto.gov.br/ccivil_03/leis/18069.htm>. Acesso em: 28 fev. 2021.

CARLOS, D. M. et al. "I don't have it, I didn't have it": experiences of families involved in violence against children and adolescentes. Rev. Bras. Enferm., Brasília, n. 73, suppl. 4, may 2020. DOI: https://doi.org/10.1590/0034-7167-2019-0195. Available on:

<https://www.scielo.br/j/reben/a/k836VpCZRsnQWM6WWN9sKHK/?lang=en>. Access in: 11 feb. 2021

FUNDO DAS NAÇÕES UNIDAS PARA A INFÂNCIA. Direitos negados: A violência contra a criança e o adolescente no Brasil, $2^{a}$ ed., Brasília, DF: UNICEF, 2006. 16-17/141-146 p. Disponível em:

<https://www.unicef.org/brazil/media/4021/file/Direitos_Negados.pdf〉. Acesso em: 12 mai. 2021.

FERREIRA, C. L. S.; CÔRTES, M. C. J.; GONTIJO, E. D. Promoção dos direitos da criança e prevenção de maus tratos infantis. Ciênc. Saúde coletiva, Rio de Janeiro, n. 24, v. 11, p. 3997-4008, nov. 2019. DOI: https://doi.org/10.1590/1413-812320182411.04352018. Disponível em: <https://www.scielo.br/j/csc/a/qxhbH35c96Dpj6RQSkYmWFH/?lang=pt>. Acesso em: 26 mai. 2021.

HABIGZANG, L. F. et. al. Grupoterapia cognitivo-comportamental para crianças e adolescentes vítimas de abuso sexual. Revista de Saúde Pública, São Paulo, n. 43, suppl. 1, p. 70-78, ago. 2009. DOI: https://doi.org/10.1590/S003489102009000800011. Disponível em: <https://www.scielo.br/j/rsp/a/xhqFSy95Nsrc4gJxFdjWhXk/abstract/?lang=pt>. Acesso em: 4 fev. 2021.

HABIGZANG, L. F. et al. Fatores de risco e de proteção na rede de atendimento a crianças e adolescentes vítimas de violência sexual. Psicologia Reflexão e Crítica, São Paulo, n. 19, v. 3, p. 379-386, 2006. DOI: https://doi.org/10.1590/S0102-

79722006000300006. Disponível em:

<https://www.scielo.br/j/prc/a/mkmzQRTLrhQzxk5hnmKhVrn/abstract/?lang=pt>. Acesso em 27 mai. 2021. 
HINO, P. et al. Interfaces of vulnerability dimensions in violence against children. Rev. Bras. Enferm., São Paulo, n. 72, suppl. 3, p. 343-347, dec. 2019. DOI: https://doi.org/10.1590/0034-7167-2018-0463. Available on: <https://www.scielo.br/j/reben/a/hDhLZt5KShRBZ9q9ghQWk5t/abstract/?lang=en>. Access in: 28 jan. 2021.

HOFFMANN, E. V. et. al. Mental health of children who work on the streets in Brazil after enrollment in a psychosocial program. Soc. Psychiatry Psychiatr. Epidemiol., s./l., n. 52, v. 1, p. 55-63, nov. 2016. DOI: https://doi.org/10.1007/s00127-016-1316-2. Available on: <https://pubmed.ncbi.nlm.nih.gov/27866219/>. Access in: 14 feb. 2021.

KATAGUIRI, L. G. et al. Characterization of sexual violence in a state from the southeast region of Brazil. Texto Contexto - Enferm., Florianópolis, n. 28, s./v., s./p., 2019. DOI: https://doi.org/10.1590/1980-265X-TCE-2018-0183. Available on: <https://www.scielo.br/j/tce/a/XP6GktxgQwNb4xdkYR4nCsG/?lang=en>. Access in: 21 apr. 2021.

MACEDO, D. M. et al. Characterization of Child Maltreatment Cases Identified in Health Services. Paidéia (Ribeirão Preto), Ribeirão Preto, n. 30, s./v., s./p., 2020. DOI: https://doi.org/10.1590/1982-4327e3018. Available on: <https://www.scielo.br/j/paideia/a/tn7mw3mgkqKgCXbGZMj4gLF/?lang=en>. Access in: 16 feb. 2021.

MELLO, A. F. et al.. Exposure to maltreatment and urban violence in children working on the streets in São Paulo, Brazil: factors associated with street work. Rev. Bras. Psiquiatr., São Paulo, n. 36, v. 3, p. 191-198, jul-sep., 2014. DOI: https://doi.org/10.1590/15164446-2013-1185. Available on:

<https://www.scielo.br/j/rbp/a/g3BZ9bKR88M3jmQ7FM7xHpK/abstract/?lang=en>. Access in: 12 jan. 2021.

MONTEZUMA, M. A.; PEREIRA, R. C.; MELO, E. M. Abordagens da alienação parental: proteção e/ou violência? Physis: Revista de Saúde Coletiva, Rio de Janeiro, n. 27, v. 4, p. 1205-1224, oct-dec. 2017. DOI: https://doi.org/10.1590/S010373312017000400018. Disponível em: $<$ https://www.scielo.br/j/physis/a/Hqqt9bcQVjBYfCnSQxpCbsN/?lang=pt>. Acesso em: 25 jan. 2021.

MORAES, C. L. et al.. The intertwined effect of lack of emotional warmth and child abuse and neglect on common mental disorders in adolescence. DOI:

https://doi.org/10.1016/j.chiabu.2018.07.008. Child Abuse \& Neglect, s./l., n. 83, s./n., p. 74-82, sep. 2018. Available on:

<https://www.sciencedirect.com/science/article/abs/pii/S0145213418302771>. Access in: 15 may 2021.

NUNES. A. C. P. et al. Violência infantil no Brasil e suas consequências psicológicas: uma revisão sistemática. Braz. J. of Develop., São José dos Pinhais-PR, n. 6, v. 10, p. 79408-79441, out. 2020. DOI: https://doi.org/10.34117/bjdv6n10-392. Disponível em: <https://www.brazilianjournals.com/index.php/BRJD/article/view/18453/14870>. Acesso em: 7 jan. 2021. 
OLIVEIRA, N. F. et al. Violência contra crianças e adolescentes em Manaus, Amazonas: estudo descritivo dos casos e análise da completude das fichas de notificação, 2009-2016. Epidemiol. Serv. Saúde, Brasília, n. 29, v. 1, s./p., 2020. DOI: https://doi.org/10.5123/S1679-49742020000100012. Disponível em:

<https://www.scielo.br/j/ress/a/Bn7BXPdTchdZzKHt4bZRYnQ/?lang=pt>. Acesso em: 10 mai. 2021.

ORGANIZAÇÃO MUNDIAL DA SAÚDE. Relatório Mundial sobre Violência e Saúde. Genebra, 2002. 5 p. Disponível em: https://portaldeboaspraticas.iff.fiocruz.br/wp-content/uploads/2019/04/14142032relatorio-mundial-sobre-violencia-e-saude.pdf. Acesso em: 14 fev. 2021.

PASIAN, M. S. et al.. Negligência infantil: A Modalidade Mais Recorrente de MausTratos. Pensando Famílias, Porto Alegre, n. 17, v. 2, p. 61-70, dez. 2013. Disponível em: <http://pepsic.bvsalud.org/scielo.php?script=sci_arttext\&pid=S1679494X2013000200005>. Acesso em: 17 abr. 2021.

PENSO, M. A. et al. Boy sexual victimization: Characteristics of family configuration and of the offender. Psic.: Teor. e Pesq., Brasília, n. 35, s./v., s./p., 2019. DOI: https://doi.org/10.1590/0102.3772e35428. Available on: <https://www.scielo.br/j/ptp/a/49CLM4NvXhqZ37bKXzf4tJf/abstract/?lang=en>. Access in: 25 feb. 2021.

PIRES, T. O.; SILVA, C. M. F. P.; ASSIS, S. G. Ambiente familiar e transtorno de déficit de atenção e hiperatividade. Revista de Saúde Pública, São Paulo, n. 46, v. 4, p. 624-632, ago. 2012. DOI: https://doi.org/10.1590/S0034-89102012005000043. Disponível em: <https://www.scielo.br/j/rsp/a/yDfwgymmXn36qkdHQXmRWCw/?lang=pt>. Acesso em: 25 jan. 2021.

PLATT, V. B. et al. Sexual violence against children: authors, victims and consequences. Ciência \& Saúde Coletiva, Rio de Janeiro, v. 23, n. 4, p. 1019-1031, apr. 2018. DOI: https://doi.org/10.1590/1413-81232018234.11362016. Available on: <https://www.scielo.br/j/csc/a/hTR8wBZKQNrYLm4HB6p849c/?lang=en>. Access in: 11 may 2021.

PLATT, V. B.; GUEDERT, J. M.; COELHO, E. B. S. Violence against children and adolescents: notification and alert in times of pandemic. Rev. paul. pediatr., São Paulo, v. 39, s./n., s./p., oct. 2020. DOI: https://doi.org/10.1590/0102.3772e35428. Available on: <https://www.scielo.br/j/rpp/a/Ghh9Sq55dJsrg6tsJsHCfTG/?lang=en> . Access in: 23 jan. 2021.

QUADROS, M. N. et al. Situação da violência contra crianças e adolescentes no Brasil. Enfermería Global, s./l., n. 44, s./v., p. 174-185, out. 2016. Disponível em: <https://scielo.isciii.es/pdf/eg/v15n44/pt_docencia2.pdf>. Acesso em: 24 jan. 2021.

RECHEINHIM, M. E.; HASSELMANN, M. H.; MORAES, C. L. Consequências da violência familiar na saúde da criança e do adolescente: contribuições para a elaboração de propostas de ação. Ciência \& Saúde Coletiva, Rio de Janeiro, n. 4, v. 1, p. 109-121, 1999. DOI: https://doi.org/10.1590/S1413-81231999000100009. 
Disponível em:

<https://www.scielo.br/j/csc/a/Yjg3SbjWYFnTfSXPbRc48rm/abstract/?lang=pt>.

Acesso em: 10 fev. 2021.

RIBEIRO, M. A.; FERRIANI, M. G. C.; REIS, N. R. Violência sexual contra crianças e adolescentes: características relativas à vitimização nas relações familiares.

Cadernos de Saúde Pública, Rio de Janeiro, n. 20, v. 2, p. 456-464, abr. 2004. DOI: https://doi.org/10.1590/S0102-311X2004000200013. Disponível em:

<http://www.scielo.br/scielo.php?script=sci_arttext\&pid=S0102-

311X2004000200013>. Acesso em: 10 mai. 2021.

SILVA, A. J. C. da; TRINDADE, R. F. C. da; OLIVEIRA, L. L. F. de. Presumption of sexual abuse in children and adolescents: vulnerability of pregnancy before 14 years.

Rev. Bras. Enferm., Brasília, n. 73, suppl. 4, s./p., 2020. DOI:

https://doi.org/10.1590/0034-7167-2019-0143. Available on:

<https://www.scielo.br/j/reben/a/Kzh6wbDhSVZhDFvqMspCDMB/abstract/?lang=en> . Access in: 12 jan. 2021.

SILVA, W. S. et al. Factors associated with child sexual abuse confirmation at forensic examinations. Ciência \& Saúde Coletiva, Salvador, v. 23, n. 2, p. 599-606, feb. 2018. DOI: https://doi.org/10.1590/1413-81232018232.04932016. Available on:

<http://www.scielo.br/scielo.php?script=sci_arttext\&pid=S1413-

$81232018000200599>$. Access in: 30 jan. 2021.

SOUZA, A. L. P. de et. al. Characterization of suicidal behavior among children in a depressive episode: case series study. Trends Psychiatry Psychother., Porto Alegre, n. 41, v. 4, p. 394-400, oct-dec. 2019. DOI: https://doi.org/10.1590/2237-6089-20180111. Available on:

<https://www.scielo.br/j/trends/a/KB8gWmZBcMnyn7LqvSWXhbK/?lang=en>. Access in: 29 apr. 2021.

WORLD HEALTH ORGANIZATION. Status report on preventing violence against children, Geneva, 2020. 1-11 p. Available on:

<https://www.who.int/publications/i/item/9789240004191>. Access in: 11 jan. 2021.

Recebido em: 01/10/2021

Aprovado em: 25/10/2021

Publicado em: 02/11/2021 